\title{
Time and Season Incidence of Complicated Colorectal Cancer.
}

\author{
Iordanov E. ${ }^{1}$, Uzunova V. ${ }^{1}$, Uchikov P. ${ }^{1}$, Podnov L. ${ }^{1}$, Sakakushev A. ${ }^{3}$, \\ Sakakushev B. ${ }^{2}$ \\ https://doi.org/10.32391/ajtes.v4i1.76
}

\begin{abstract}
Colorectal cancer complications are seldom analyzed for occurrence through day and night, working days and weekends, as well as throughout seasons. We hypothesized that information and analysis of high/low complicated colon cancer incidence in the above mentioned terms can improve our preparedness and manage better the resources for superior outcomes. Therefore, we performed a retrospective study during $2010-2018$ timeframe consisting of 165 patients with complicated colorectal cancer operated on emergently or urgently in the Second Clinic of General and Thoracic Surgery, University Hospital St George, Plovdiv, Medical University Plovdiv, Bulgaria.

Keywords: Complicated Colon Cancer (CCC), Emergency General Surgery (EGS), After Hours Surgery (AHS), Weekend Effect (WE)
\end{abstract}

*Corresponding Author: Emanuil Iordanov

=凶 E-mail: emo.iordanov@abv.bg

1Second Clinic of General and Thoracic Surgery, University Hospital St George, Plovdiv, Medical University Plovdiv, Bulgaria

2First Clinic of General Surgery, University Hospital St George, Plovdiv, Medical University Plovdiv, Bulgaria

3Wageningen Economic Research, Wageningen University \& Research, The Netherlands 


\section{Full Text}

\section{Introduction}

The provision and delivery of EGS services is a globally and regionally important issue ${ }^{1}$. Seasonal variation in medical conditions is well established; however, its impact on EGS cases remains unclear ${ }^{2}$. An association between weekend health care delivery and poor outcomes has become known as the "weekend effect." Evidence for such an association among surgery patients has not previously been synthesized ${ }^{3}$. Recently, there has been much debate over the existence of a "weekend effect", accounting for excess mortality in those patients treated at the weekend ${ }^{4}$. Increasing number of publications recently show the danger of after-hours medical activity. Medical errors occur more often at night when physicians appear to be less 5 .

Realizing the significance complicated colon cancer surgery, we aimed at analyzing its rate and distribution throughout day/night hours, week days (on-off) and seasons, hypothesizing that its occurrence and time of management may influence outcomes.

\section{Methods}

We performed a retrospective study of 165 patients with complicated colorectal cancer operated on emergently or urgently between 2010 - 2018 in the Second Clinic of General and Thoracic Surgery, University Hospital St George, Plovdiv, Medical University Plovdiv, Bulgaria. Patient's files were searched for admission and operation data throughout day hours (7.30-19.30), night shifts (19.30-7.30); working week days (Monday - Friday); weekends (Saturday - Sunday) and seasons - Spring (March, April, May), Summer (June, July, August), Fall (September, October, November), and Winter (December, January, February). Additionally rate, distribution and proportion of complications like perforation, bowel obstruction and "others" have been studied for the abovementioned time spans. Relevant literature search was performed to compare the results obtained, discuss and formulate statements for conclusions and optimal propositions.

\section{Results}

Analyzing our database, we found out that the occurrence of complicated colon cancer (CCC) throughout the day (7.30 a.m. - 7.30.p.m. ) was $73.94 \%(\mathrm{n}=112)$ and $43 \%(\mathrm{n}=26.6)$ in the night (Figure 1). 
No. of patients by time of patient entry

(2010 - 2018)

43

122

- Day - Night

Figure 1: CCC Patients rate by day/night

During the week days CCC showed the $\mathrm{n}=26(16 \%)$, Friday $-\mathrm{n}=23(14 \%)$ and following incidence - Monday $-\mathrm{n}=23$ Sunday - $\mathrm{n}=23$ (14\%) ( Figure 2). $(14 \%)$, Tuesday $-\mathrm{n}=33(20 \%)$, Wednesday $-\mathrm{n}=23(14 \%)$, Thursday -

No. of patients by weekday (2010-2018)

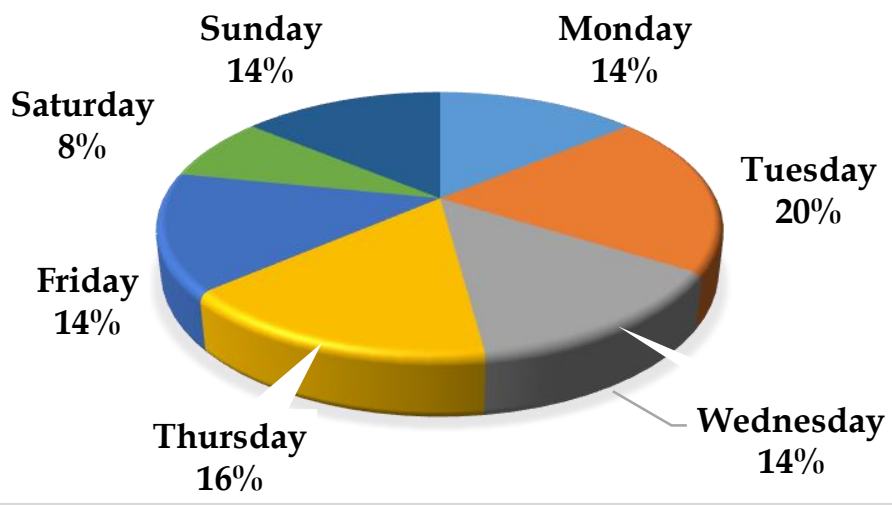

Figure 2: CCC patient rate per days in the week.

The season's occurrence of CCC was $33.94 \%(n=56)$ in winter, $26.06 \%(n=43)$ in spring, $22.42 \%(\mathrm{n}=37)$ - in summer and
$17.58 \%(\mathrm{n}=29)$ - in autumn respectively (Figure 3). 


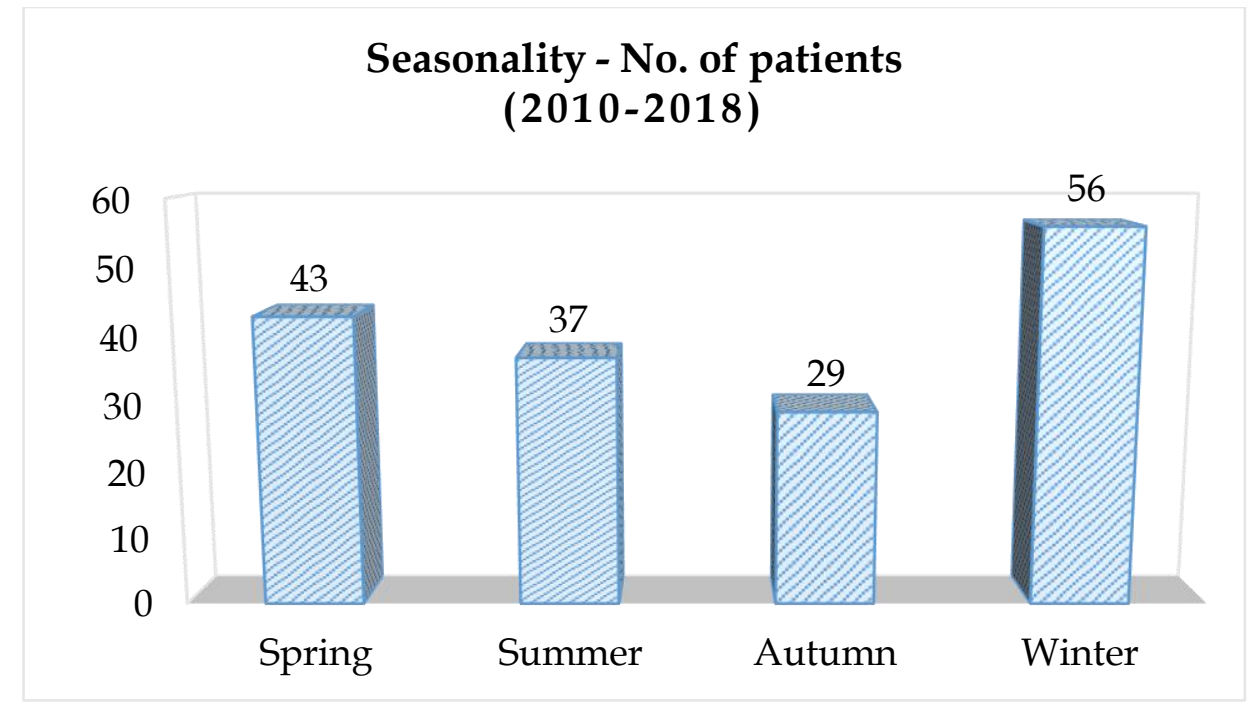

Figure 3: CCC patient rate in seasons.

Bowel obstruction occurred in $67.27 \%$ $(\mathrm{n}=111)$, peritonitis $-18.79 \%(\mathrm{n}=31)$, followed by all the other complications - $13.94 \%$ (n 23) (Figure 4).

\section{No. of patients by major complication} (2010-2018)

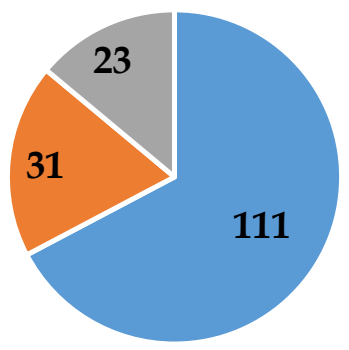

- Ileus $\quad$ Peritonitis $\quad$ Other

Figure 4: Overall CC patients' rate of major complications.

Complications throughout the day (7.30. a.m. - 7.30. p.m.) and night time (7.30 p.m. - 7.30 a.m.) were as follows: bowel obstruction during day time $-52.12 \%$ $(\mathrm{n}=86)$, at night $-15.15 \%(\mathrm{n}=26)$; peritonitis by day/night $-10.91 \%$ (n 18) and $7.88 \%$ (n 13) respectively (Figure 5). 


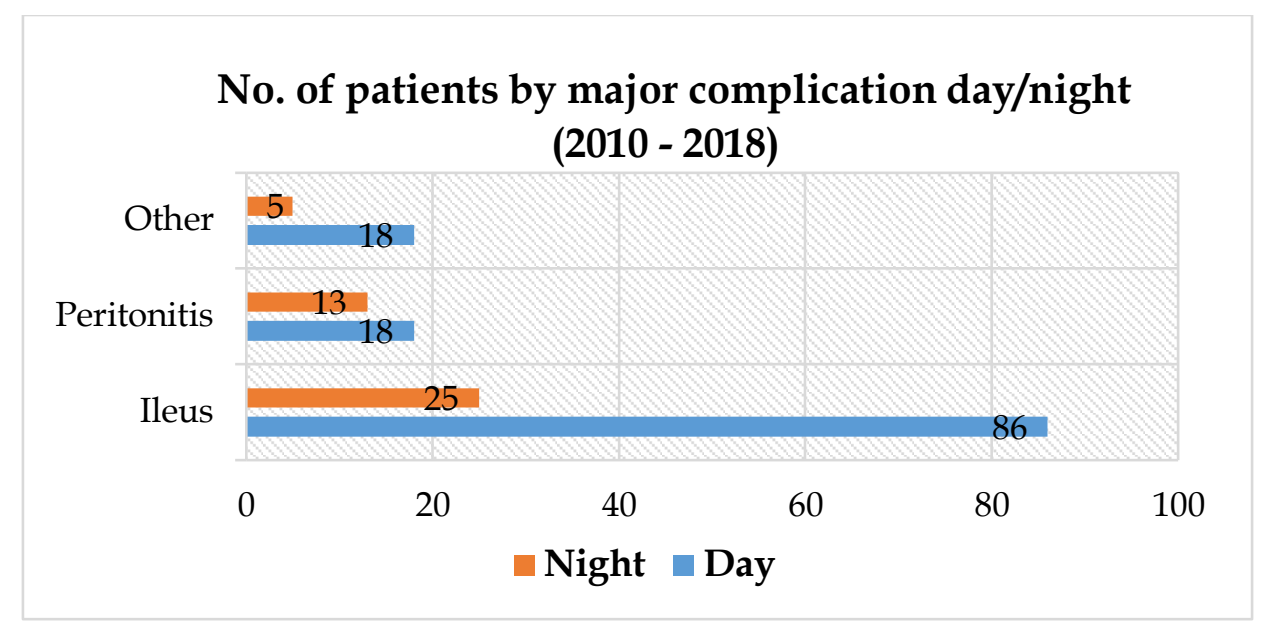

Figure 5: CC patients' rate of major complications per day/night.

The rate of major CCC was highest in the middle of the week, where, the overall bowel obstruction incidence in daytimes accounted for $35.15 \%(n=58)$ of all types of complications and on Saturday was 5.45\% (n=9) (Figure 6).

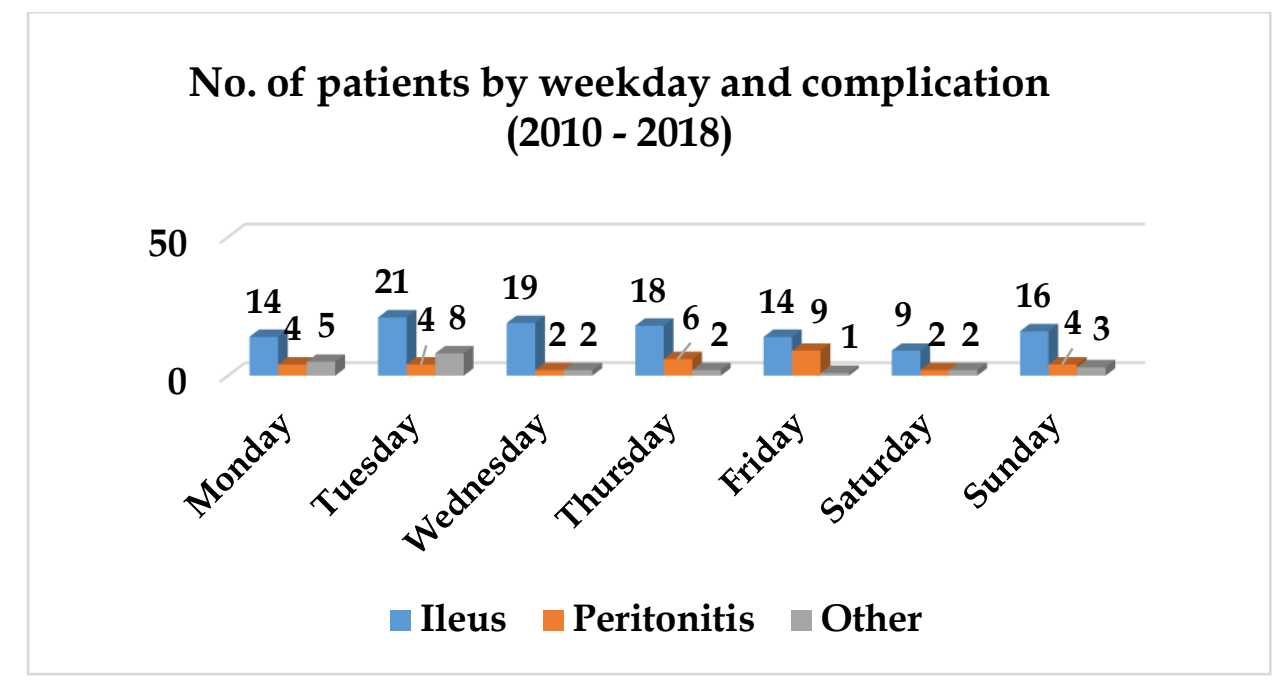

Figure 6: CC patient's rate of major complications per days of the week.

Peritonitis had $4.85 \%(\mathrm{n}=8)$ rate on Tuesday, 3.03\% $(n=5)$ - on Monday, $1.81 \%(\mathrm{n}=3)$ on Sunday and $1.21 \%(\mathrm{n}=2)$ on Wednesday, Thursday and Saturday respectively (Figure 6). 
Colorectal cancer complication rate per season was relatively similar, where the dominating - bowel obstruction, in the year's four seasons raised from $13.33 \%$ (n 22) in autumn up to $19.39 \%$ (n 32) in spring (Figure 7).

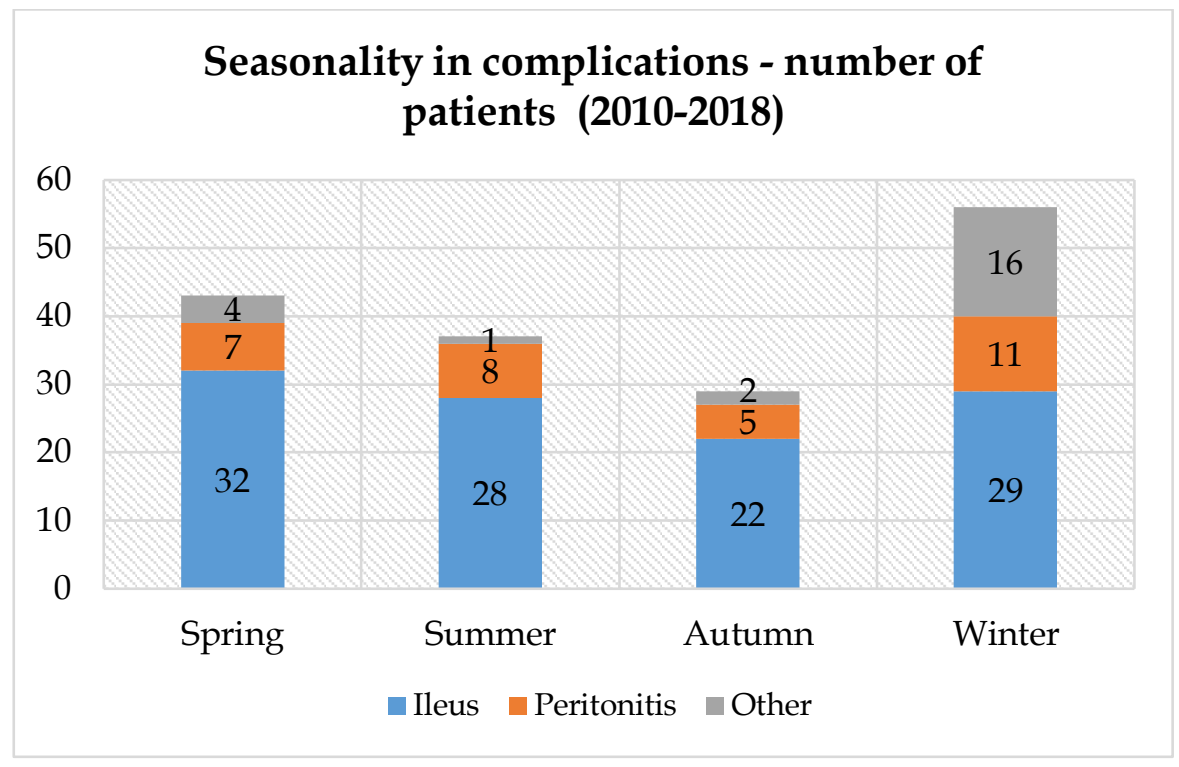

Figure 7: CC patient's rate of major complications per season.

Peritonitis rate in winter was $9.70 \%$ $(\mathrm{n}=16)$, in spring $-2.42 \%(\mathrm{n}=4)$, in autumn $-1.21 \%(\mathrm{n}=2)$ and in summer $0.61 \%(\mathrm{n}=1)$.

\section{Discussion}

Patient's history and physical examination have been the foundation of medical diagnosis for centuries. However, as laboratory tests and diagnostic imaging has expanded, physical examination skills have been deemphasized in medical education, and clinicians have become more reliant on tests and imaging ${ }^{6}$.

Data from 1484116 EGS hospital episodes of adults (aged >15) in Scotland, between 1997 and 2016 showed that admissions have increased over time, whereas that of EGS operations have decreased over time. Male admissions were unchanged, but with fewer operations over time, whereas female admissions increased significantly over time with no change in the operation rate. These data help inform stakeholders in EGS service planning and delivery, as well as in surgical training, what has occurred in recent history ${ }^{1}$.

It is unclear whether the time of day for emergency surgery is associated with postoperative mortality. The assessment of 9319 patients who had emergency surgery as their first surgery at the Jewish General Hospital, Montreal, QC, Canada from April 2010 to March 2015 
demonstrated that there was no significant association of time of day with postoperative mortality, with adjusted OR $(95 \% \mathrm{CI})$ of $1.61(0.96-2.72)$ for night vs. day, $\mathrm{p}=0.07 ; 1.29(0.78-2.13)$ for night vs. evening, $\mathrm{p}=0.33$; and 1.26 (0.89-1.78) for evening vs. day, $\mathrm{p}=0.20^{7}$. More and more publications show the danger of after-hours medical activity. Medical decision making (Dg) may be affected at night, as Singh et al. showed increased rates of unknown diagnoses and a trend toward increased false negative appendectomies in the setting of acute appendicitis ${ }^{8}$.

Evidence suggests that sleep deprivation associated with night-time working may adversely affect performance resulting in a reduction in the safety of surgery and anesthesia ${ }^{9}$. There is little evidence, as yet, to inform the issue of the effect of fatigue on surgical performance ${ }^{10}$.

Gray et al. showed after-hours surgery to be related to higher complications ${ }^{11}$.

In a series of 739 patients with primary colorectal anastomosis performed between1997-2007. Komen \& alt. fund out that patients operated upon afterhours had more than a twofold increased risk of anastomotic leakage, independent of the urgency of the operation, which may be explained by decreased technical performance of the operating team at night ${ }^{12}$.

Orthopedic trauma surgery performed in the afternoon or at night had significantly higher mortality rates as well as general, but not intra- or postoperative complication rates vs. orthopedic trauma surgery performed in the morning. It is critical to maximize patient safety and provide the best possible care for patients at any time of the day. This includes surgeon selfawareness and carefully choosing when to operate ${ }^{13}$.

In prospective cohort study(European Surgical Outcome Study) of11290 patients undergoing urgent surgery from 498 hospitals in 28 European countries the 636(5.6\%) in-hospital mortality van Zaane \& alt. did not identify any relationship with the time of day at which the procedure was performed 9 .

Our cases CCC rate was approximately three times higher $(n=112 / 73.94 \%)$ throughout the day (7.30 a.m. - 7.30.p.m) compared to the night rate $(n=26.6 / 43 \%)$. In their systematic review Parker \& alt., recommends implementing on-call periods of no more than 12 hours in duration, with adequate rest periods every 24 hours ${ }^{14}$.

Variation in weekly patterns reflected in some surgical studies found evidence of poorer morbidity and mortality outcomes from procedures performed overnight. It is notable however, that studies examining variation in care are often naturally focused on morbidity and mortality, while there is little evidence regarding the diagnostic accuracy of procedures performed outside of regular working hours. 
We decided to test the hypothesis that diagnostic accuracy would be reduced in the off hours setting, given the aforementioned challenges.

Concha et al. suggested that the perceived suboptimal quality of care may be caused by reduced staffing levels or that patients who present outside of regular working hours have a greater burden of comorbidities with a worse pre-morbid ${ }^{15}$.

Freemantle et al. described a $16 \%$ increase in 30-day mortality between patients admitted on a Sunday compared to a Wednesday ${ }^{16}$.

In our series we registered a dominance in the middle of the week $/ 50 \%$ of all on Tuesday, Wednesday and Thursday. The highest in rate of CCC day of the week was Tuesday $-\mathrm{n}=33(20 \%)$, the lowest Saturday $-\mathrm{n}=13(8 \%)$.

Surgery is no exception to this and similar results have been reported in both the elective and non-elective surgical caseloads in studies focusing on procedures performed on Saturdays or Sundays 17,18 .

Laparotomy, adhaesiolys is, partial colectomy, and small bowel resection $/ p<0.01$ ), had higher mortality for weekend cases while appendectomy, cholecystectomy, and peptic ulcer disease repair did not. Among the four procedures with increased weekend mortality, patients undergoing weekend procedures also had increased severity of illness and shorter time from admission to surgery $(p<0.01)$. Multivariate analysis adjusting for patient characteristics

demonstrated independently higher mortality on weekends for these same four procedures $(p<0.01)^{19}$.

In a study of $1,344,828$ individual patient records (6.7 million weighted admissions). Metcalfe \& alt. found evidence that outcomes are marginally worse for EGS patients admitted at weekends ${ }^{20}$. In a study of data for emergency general surgical admissions to National Health Service hospitals in the Northern Deanery in England between 2000 and 2014, McCallum \& alt., analyzing 12100 in-hospital deaths within 30 days of admission (3.3 per cent) the weekend effect describes excess mortality associated with hospital admission on Saturday or Sunday ${ }^{21}$. They concluded that during the past 15 years there has been a weekend effect in patients undergoing emergency general surgery based on day of operation, but not day of admission. Overall mortality for emergency general surgery has improved significantly, and in the past 5 years the increased mortality risk of weekend surgery has reduced. In their systematic review and metaanalysis of 29 studies including over 8 million patients, Smith \& alt. demonstrate that mortality are higher after admission for urgent or emergent surgery on the weekend relative to weekdays. These results are consistent with the presence of a weekend effect in surgical care. Future studies should 
focus on clarifying the contributing factors to poor outcomes and developing strategies to potentially improve safety and mitigate adverse events associated with weekend surgical care ${ }^{3}$.

The National Inpatient Sample (NIS) database over an 8-year period (20042011) evaluated 63,911,033 admission records were of which 493,569 were appendicitis, 395,838 were cholecystitis, and 412,163 were diverticulitis, where seasonal variation was confirmed in EGS $(F=159.12, P<0.0001)$ admissions. In the sub-analysis, seasonal variation was found in acute appendicitis $(F=119.62, P$ $<0.0001)$, acute cholecystitis $(F=37.13, P$ $<0.00011^{2}$. Further study is needed to fully describe the etiologies of the seasonal variation in outcomes ${ }^{22}$.

The CCC rate of our patients in different seasons demonstrated a steady and even fall from winter - $33.94 \% \quad(n=56)$, to spring $-26.06 \%(n=43)$, summer $-22.42 \%$ $(\mathrm{n}=37)$ and autumn $-17.58 \%(\mathrm{n}=29)$.

Autumn and winter showed almost twofold increase of manifestation of CCC, where bowel obstruction occurred 3 times more common $(67.27 \% / \mathrm{n} 111)$ than peritonitis $(18.79 \% / \mathrm{n}=31)$, followed by all the other complications $13.94 \% / n=23$.

Complications throughout the day (7.30. a.m. - 7.30. p.m.) and night time (7.30 p.m. - 7.30 a.m.) in our series revealed considerable dominance of bowel obstruction during day time $52.12 \%(n=86)$ - more than half of all complications whatsoever, while peritonitis showed almost similar incidence by day /night $-10.91 \% \quad(n=18)$ and $\quad 7.88 \% \quad(\mathrm{n}=13) \quad$ respectively. Throughout the week, bowel obstruction incidence in daytimes accounted for $35.15 \% \quad(n=58) \quad$ of all types of complications and was less common on Saturday - 5.45\% $\quad(n=9)$. Peritonitis demonstrated more even distribution in the week, with highest rate on Tuesday $4.85 \%(\mathrm{n}=8)$, less on Monday - 3.03\% $(n=5)$ and similar incidence in the other week days, varying from $1.21 \%(n=2)$ to $1.81 \%(\mathrm{n}=3)$.

Per season CCC demonstrated relatively similar rate of the dominating - bowel obstruction, occurring with slight deviation throughout the year's four seasons varying from $13.33 \%(n=22)$ in autumn up to $19.39 \%(n=32)$ in spring. Peritonitis occurred most often in winter $-9.70 \%(n=16)$, four times less in spring $2.42 \%(\mathrm{n}=4), 1.21 \%(\mathrm{n}=2)$ in autumn and only $0.61 \%(\mathrm{n}=1)$ in summer.

Back in 1975, Hampton JR, et al. suggested that a protocol approach to pre-operative workup and management, which may ensure benefit for difficult to assess patients, as well as standardization of the decision when to operate ${ }^{23}$. Studies on more patients and more factors, with longer follow-up, will probably clarify important associations of time of emergency surgery with postoperative mortality and morbidity, as well as operating overnight relative to 
daytime for emergency ${ }^{7}$. Further studies can identify modifiable risk factors in after-hours surgery ${ }^{13}$, or the impact of staff fatigue in day, evening, night and more prolonged periods of sleep deprivation on surgeons' performance ${ }^{24}$. Hospital admissions for Emergency General Surgery (EGS) have increased $28 \%$ since 2001, accounting over 27 million annually, where EGS procedures bare up to 8 times higher lethal risk, than elective ones. EGS admissions and costs are projected to increase $45 \%$ to $\$ 41.20$ billion annually by 2060, using US Census projections. EGS care should be considered a national priority, where to optimize outcomes, consistent definitions, risk assessment, case registry and standardized patient care, using evidence-based guidelines and bundles is recommended 25 .

\section{Conclusions}

The "after hours effect" often related to complications and worse results, substantially depends on initial diagnosis in the off hours setting with decreased resources, resulting in suboptimal management.

A protocoled approach to pre-operative workup and management, especially for difficult to assess patients, as well as standardization of indications and timing of surgery based on day/night, week days and season rate of occurrence and type of major complications of complicated colorectal cancer cases could be a beneficial tool to optimize final outcome.

\section{References}

1. Wohlgemut, J., Ramsay G., Jansen, J. The Changing Face of Emergency General Surgery. A 20-year Analysis of Secular Trends in Demographics, Diagnoses, Operations, and Outcomes Annals of Surgery: September 26, 2018

2. Zangbar B., Rhee, P., Pandit V. Seasonal Variation in Emergency General Surgery Annals of Surgery. 263(1):76-81， January 2016.

3. Smith S., Yamamoto J., Roberts D., $\&$ alt. Weekend Surgical Care and Postoperative Mortality: A Systematic Review and MetaAnalysis of Cohort Studies. Med Care. 2018 Feb; 56(2):121-129.

4. Godlee F. The "weekend effect". BMJ. 2016;353:i2801

5. Catchpole K. et al (2008) Teamwork and error in the operating room: analysis of skills and roles. Ann Surg. 247(4):699706.

6. Schultz MA ${ }^{1}$, Doty M. Why the history and physical examination still matter. JAAPA. 2016 Mar; 29 (3):41-5. 
7. Tessler M., Charland L., Wang N., Correa J. The association of time of emergency surgery - day, evening or night - with postoperative 30-day hospital mortality. Anaesthesia.2018 Nov; 73(11):1368-1371.

8. Singh K., Wilson M. and Coats M. Does time of surgery influence the rate of false-negative appendectomies? A retrospective observational study of 274 Patients. Patient Safety in Surgery (2018) 12:33

9. van Zaane B., van Klei W., Buhre W., \& alt. European Surgical Outcomes Study (EuSOS) group for the Trials groups of the European Society of Intensive Care Medicine and the European Society of Anaesthesiology /Collaborators (1937). Nonelective surgery at night and inhospital mortality: Prospective observational data from the European Surgical Outcomes Study. Eur J Anaesthesiol. 2015 Jul; 32(7):477-85.

10. Sturm L., Dawson D., Vaughan R. $\&$ alt. Effects of fatigue on surgeon performance and surgical outcomes: a systematic review. ANZ J Surg. 2011 Jul-Aug; 81(78):502-9.

11. Gray A. (2000) United Kingdom national confidential enquiry intoperioperative deaths. Minerva Anestesiol. 66(5):288-292

12. Komen N., Dijk J-W., Lalmahomed Z. et al. After-hours colorectal surgery: a risk factor for anastomotic leakage. Int. J. Color. Dis.2009; 24(7):789-95.

13. Halvachizadeh et al. Does the time of day in orthopedic trauma surgery affect mortality and complication rates? Patient Safety in Surgery (2019) 13:8

14. Parker, RS, Parker, P. The impact of sleep deprivation in military surgical teams: a systematic review. Journal of the Royal Army Medical Corps 2017; 163: 158- 63

15. Concha OP, et al. Do variations in hospital mortality patterns after weekend admission reflect reduced quality of care or different patient cohorts? A population-based study. BMJ Qual. Saf. 2014;23(3):215-22

16. Freemantle N, et al. Weekend hospitalization and additional risk of death: an analysis of inpatient data. J R Soc. Med. 2012; 105(2):74-84.

17. Aylin P, et al. Day of week of procedure and 30 day mortality for elective surgery: retrospective analysis of hospital episode statistics. BMJ. 2013; 346.

18. Worni M., et al. Worse outcomes in patients undergoing urgent surgery for left-sided 
diverticulitis admitted on weekends vs. weekdays: a population based study of 31832 patients. Arch Surg. 2012; 147(7):649-55.

19. Hoehn R., Go D., Dhar V. \& alt. Understanding the "Weekend Effect" for Emergency General Surgery. J Gastrointest. Surg. 2018 Feb; 22(2):321-328.

20. Metcalfe D, Castillo-Angeles M, Rios-Diaz \& alt. Is there a "weekend effect" in emergency general surgery? J Surg Res. 2018 Feb; 222:219-224.

21. McCallum I. McLean R., Dixon S., O' Loughlin P. Retrospective analysis of 30-day mortality for emergency general surgery admissions evaluating the weekend effect. Br J Surg. 2016 Oct; 103(11):1557-65.

22. Englesbe M., Pelletier S., Magee J., et al. Seasonal variation in surgical outcomes as measured by the American College of Surgeons-National Surgical Quality Improvement Program (ACSNSQIP).Ann Surg. 2007;246(3):456-62 discussion 635

23. Hampton JR, et al. Relative contributions of history-taking, physical examination, and laboratory investigation to diagnosis and management of medical outpatients. Br Med J. 1975;2(5969):486-9

24. Kamal Y. Time of day and 30-day mortality after emergency surgery, Anaesthesia, 74, 2, 258258, 2019

25. Havens, J., Neiman, P; Campbell, B. The Future of Emergency General Surgery. Annals of Surgery: December 29, 2018 